\title{
Autoreporte de salud y enfermedad en la adolescencia: Cuestionario CHIP-AE en adolescentes escolarizados chilenos
}

\author{
ALFONSO URZÚA M. ${ }^{1}$ \\ 1. Psicólogo, Licenciado en Psicología, Magíster en Salud Pública, Doctor en Psicología Clínica y de la Salud. \\ Escuela de Psicología, Universidad Católica del Norte, Chile.
}

\begin{abstract}
Health and illness selfreport in adolescence: CHIP-AE questionnaire in chilean school adolescents
\end{abstract}

Objective: To describe and analyze values obtained from the application of the Child Health and Illness Profile-Adolescent Edition (CHIP-AE) in school chilean adolescents. Method: The questionnaire was applied to a sample of 531 school adolescents of Antofagasta (249 were men and 282 women), with a mean of age of 15.44 years. Means and deciles for each of the domains and subdomains of the instrument, separated by sex and range of age were calculated. Results: Men presented significantly highest median level of satisfaction and resistance $(\mathrm{p}<0.01 \mathrm{y} \mathrm{p}<0.05)$ and women highest score in welfare $(\mathrm{p}<0.01)$. By ge 16 to 18 vs 13 to 15 years old group, had significantly highest scores in welfare and resistance $(p<0.001$ and $p<0,05)$, and less in risk and performance ( $\mathrm{p}<0.05$ and $\mathrm{p}<0.05$, respectively). Conclusion: Results are not different for spanish study, except on welfare were men obtained lower scores and women highest. These results can be use as comparison pattern in longitudinal studies and to compare with others populations or interventions studies in adolescent health.

(Key words: Health, adolescence, CHIP-AE, quality of life).

Rev Chil Pediatr 2012; 83 (4): 336-344

\section{RESUMEN}

Objetivo: Describir y analizar los resultados de la aplicación del cuestionario Perfil de Salud y Enfermedad Infantil, versión Adolescentes (CHIP A-E), en adolescentes escolarizados chilenos. Pacientes y Método: Se aplicó el cuestionario a una muestra de 531 adolescentes escolarizados de la ciudad de Antofagasta (249 hombres y 282 mujeres), con una media de edad de 15,44 años. Se presentan las medias y deciles para cada dimensión y subdimensiones, separadas por sexo y rango de edad. Resultados: Los hombres presentan medias significativamente más elevadas a nivel de la satisfacción y resistencia ( $\mathrm{p}<0,01$ y p $<0,05$ ), las mujeres puntúan más alto en bienestar ( $<$ 0,01). Por edad, los de 16 a 18 años vs 13 a 15 años puntúan significativamente más en bienestar $(p<0,001)$ y en resistencia $(p<0,05)$; y menos en riesgos y rendimiento $(p<0,05$ y $p<0,05$,

Recibido el 29 de mayo de 2009, devuelto para corregir el 10 de noviembre de 2010, segunda versión el 22 de diciembre de 2010, tercera versión el 24 de marzo de 2012, aceptado para publicación el 8 de junio de 2012.

Correspondencia a:

Dr. Alfonso Urzúa M.

E-mail: alurzua@ucn.cl 
respectivamente). Conclusión: Los resultados observados no difieren de lo encontrado en población española excepto en bienestar, donde los varones obtienen puntajes menores y las mujeres superiores. Estos resultados servirán de patrón de comparación para futuros estudios longitudinales o intervencion en salud adolescente, así como de otras poblaciones.

(Palabras clave: Salud, adolescencia, CHIP-AE, calidad de vida).

Rev Chil Pediatr 2012; 83 (4): 336-344

\section{Introducción}

Cuando hablamos del estado de salud de los adolescentes, generalmente se suele centrar la mirada desde el punto de vista de la morbilidad presente en dicho grupo etario. Solo en los últimos años ha habido un paulatino crecimiento e incorporación de indicadores más comprensivos de la salud que no estén centrados en la enfermedad o en la mortalidad, sino en la salud desde un prisma positivo, centrándose desde la mirada del propio adolescente, o que identifiquen las condiciones que favorecen el desarrollo saludable de éstos ${ }^{1}$. En este contexto, la percepción del estado de salud y la calidad de vida se han constituido como algunas de las áreas emergentes de desarrollo en el campo de las ciencias sociales y de la salud en los últimos años. Una de las principales líneas de trabajo en estos temas han sido la elaboración y posterior validación de instrumentos destinados a medir el constructo en población infantil y adolescente, existiendo a la fecha diversos instrumentos desarrollados principalmente en Estados Unidos ${ }^{2-4}$ y adaptados en España ${ }^{5}$. En Chile son escasos los artículos que reportan propiedades de este tipo de instrumentos en población infantil y adolescente ${ }^{6-8}$.

Pese a este reciente incremento en los artículos de investigación que reportan tanto la confiabilidad como los distintos tipos de validez de los instrumentos utilizados, son pocos los artículos que muestran los valores encontrados con un sentido práctico, es decir que permitan: a) la evaluación de los adolescentes con base al valor de su grupo poblacional de referencia; b) establecer líneas base para programas de intervención, y c) realizar estudios longitudinales sobre la salud de los adolescentes $^{9}$. En Chile, son escasos los reportes sobre autoreportes del estado de salud o de la calidad de vida en adolescentes, estando estos vinculados preferentemente al desarrollo de una enfermedad $^{10-12}$.

Uno de los instrumentos que ha evidenciado niveles adecuados de confiabilidad y validez en su versión en castellano es el Child Health and Illness Profile-CHIP AE, cuyo origen se encuentra en EE.UU. ${ }^{13,14}$ y cuyo uso se ha extendido a varios idiomas ${ }^{15,16}$. Este instrumento ha demostrado una buena capacidad para discriminar entre grupos (estado de salud, edad y sexo) y a través de su utilización se ha reportado valores poblacionales derivados de muestras en otros países ${ }^{9,17}$.

El objetivo de este artículo evaluar la autopercepción de salud y enfermedad en adolescentes chilenos escolarizados mediante el cuestionario CHIP-AE, separados por rango de edad y por sexo, y comparalos con los datos obtenidos con la versión española.

\section{Pacientes y Método}

\section{Participantes}

A fin de cuidar una adecuada participación de los adolescentes y dada la imposibilidad de tener una muestra probabilística, se consideró la opción de obtener una muestra intencionada de tipo estratificada. Bajo este parámetro, para la recolección de los datos se consideraron como elementos de estratificación el tipo de establecimiento educacional, el sexo y el rango de edad. Se seleccionaron así tres colegios particulares, tres colegios subvencionados (privado con aporte financiero estatal) y tres establecimientos públicos municipalizados. Posteriormente se realizó una segunda estratificación, a fin de asegurar una similar participación de hombres y mujeres y una tercera para equiparar ambos rangos de edad (12-15 y 
16-19 años) en cada uno de los establecimientos seleccionados.

Bajo este procedimiento se logró recoger información en 531 adolescentes, de los cuales 249 (46,9\%) fueron hombres y 282 (53,1\%) mujeres, todos de la ciudad de Antofagasta. De este total, 196 (37,2\%) estudiaban en colegios particulares, 165 (31,3\%) en colegios subvencionados y 166 (31,5\%) en establecimientos públicos municipalizados. De cada establecimiento se definieron a los alumnos según su grado escolar en: primero medio $(37,4 \%)$, segundo medio $(30,6 \%)$ y tercero medio (32\%). La edad de los encuestados osciló entre los 13 y los 18 años ( $\mathrm{M}=15,44$, $\mathrm{DE}=1,07, \mathrm{Md}=15, \mathrm{Mo}=16$ ).

\section{Instrumentos}

El perfil de salud y enfermedad el cuestionario CHIP-AE está constituido por seis dimensiones (Tabla 1): a) Satisfacción (la cual incluye la percepción de salud general y la autoestima); b) Bienestar (incorpora bienestar tanto físico como emocional, además de indagar en síntomas que pudiesen interferir en la sensación de bienestar); c) Resistencia (referida a conductas protectoras contra futuros problemas de salud); d) Riesgos (estados o conductas que aumentarían la probabilidad de que surgiese un problema de salud); e) Rendimiento (refleja las actividades sociales esperadas para un determinado grupo de edad); y f) Enfermedades (inquiere sobre la presencia de enfermedades físicas, mentales o lesiones y deficiencias definidas desde una perspectiva biomédica).

La mayor parte de los ítems incluidos en el CHIP-AE tienen opción de respuesta tipo Likert, que va desde 1 ("Completamente en desacuerdo") hasta 5 (“Completamente de acuerdo”). Puntuaciones altas indican mayor satisfacción, bienestar, resistencia, menos riesgos, menor presencia de enfermedades y más funciones. Para esta investigación se ha utilizado la versión española ${ }^{15,16}$ adaptada a la población chilena, la cual presentó una consistencia interna adecuada, con alfas de Cronbach entre 0,83 y 0,89, una adecuada estructura factorial similar a la propuesta teórica y capaz de discriminar entre rangos de edad y entre sujetos sanos y no sanos ${ }^{18}$.

El proyecto fue aprobado por el Comité de Ética de la Universidad Católica del Norte. Una vez que la investigación contó con la aprobación del Comité del área clínica de la Escuela de Psicología, así como de la Corporación Municipal de Desarrollo Social, se obtuvo el asentimiento de los alumnos voluntarios y se comenzó con el proceso de aplicación, el cual fue realizado de manera colectiva por curso, o varios cursos a la vez, dependiendo del establecimiento. De cada establecimiento se definieron cursos pertenecientes a los niveles primero, segundo y tercero medio utilizándose para esto la hora de consejo de curso o de orientación dependiendo del establecimiento. Los cuestionarios fueron llenados individualmente por todos los adolescentes que voluntariamente dieron su asentimiento para participar en la investigación, siendo el porcentaje de participación del 100\%. El tiempo promedio de llenado fue de 40 minutos. Una vez fueron recogidos los datos, estos fueron ingresados en una base de datos construida en SPSS 14.0.

Para efectos del análisis de los datos, se estandarizaron las puntuaciones medias de cada dimensión y subdimensión a una media arbitraria de 50 y una desviación estándar de 10. Esto permitirá su comparación con otras poblaciones de adolescentes cuyos datos hayan seguido el mismo procedimiento, como lo es el caso de la muestra española en el estudio de Serra-Sutton ${ }^{9}$. Para obtener cada valor se consideró el puntaje obtenido por cada participante en cada dimensión o subdimensión (rango 1 a 5), así como la media y desviación estándar del grupo total:

$$
\left[\frac{\text { puntuación de } n n \text { en } \mathrm{x} \text { - media del grupo en x x } 10}{\text { DE del grupo en } \mathrm{x}}\right]+50
$$

\section{Resultados}

\section{Participantes}

La tabla 2 muestra la distribución de los participantes en la investigación estratificados por rango de edad, por sexo y por tipo de establecimiento.

\section{Valores de Referencia}

La tabla 3 contiene los puntajes estandarizados para cada una de las dimensiones y subdimensio- 
nes del CHIP-AE separados por rango de edad y por sexo. A nivel de las dimensiones, los hombres presentan medias significativamente más elevadas a nivel de la satisfacción $\left(\mathrm{t}_{(525)}=3,48 ; \mathrm{p}<0,01\right) \mathrm{y}$ resistencia $\left(\mathrm{t}_{(525)}=2,28 ; \mathrm{p}<0,05\right)$. Las mujeres en tanto puntúan más alto en bienestar $\left(\mathrm{t}_{(525)}=-4,98 ; \mathrm{p}\right.$ $<0,01)$.

$\mathrm{Al}$ analizar por edad, los participantes del rango entre 16 a 18 años tienen una media significativamente mayor que el grupo de 13 a 15 años en bien-

Tabla 1. Dimensiones y subdimensiones del perfil CHIP-AE

\begin{tabular}{|c|c|}
\hline Dimensiones & Subdimensiones (número ítems) \\
\hline I. Satisfacción & $\begin{array}{l}\text { 1. Satisfacción con la salud general (7) } \\
\text { 2. Autoestima (5) }\end{array}$ \\
\hline II. Bienestar & $\begin{array}{l}\text { 1. Bienestar físico (24) } \\
\text { 2. Bienestar emocional (14) } \\
\text { 3. Limitaciones de actividad (7) }\end{array}$ \\
\hline III. Resistencia & $\begin{array}{l}\text { 1. Participación familiar (7) } \\
\text { 2. Resolución de problemas ( } 8 \text { ) } \\
\text { 3. Actividad física (5) } \\
\text { 4. Salud y seguridad en el hogar (11) }\end{array}$ \\
\hline IV. Riesgos & $\begin{array}{l}\text { 1. Riesgo individual (18) } \\
\text { 2. Amenazas a logros (15) } \\
\text { 3. Influencia de pares (5) }\end{array}$ \\
\hline V. Rendimiento & $\begin{array}{l}\text { 1. Rendimiento académico (7) } \\
\text { 2. Rendimiento laboral (4) }\end{array}$ \\
\hline VI. Enfermedades & $\begin{array}{l}\text { 1. Enfermedades agudas leves (10) } \\
\text { 2. Enfermedades agudas graves (9) } \\
\text { 3. Enfermedades recurrentes (11) } \\
\text { 4. Enfermedades clínicas de larga duración (6) } \\
\text { 5. Enfermedades quirúrgicas de larga duración (5) } \\
\text { 6. Enfermedades psicológicas (4) }\end{array}$ \\
\hline
\end{tabular}

Tabla 2. Distribución de la muestra total por tipo de establecimiento, sexo y rango de edad

\begin{tabular}{|lrccc|}
\hline & \multicolumn{4}{c|}{ Tipo de Establecimiento, $\mathbf{n}$} \\
\hline Público & $\begin{array}{c}\text { Subven- } \\
\text { cionado }\end{array}$ & Particular & Total \\
$13-15$ años & 31 & 51 & 52 & 134 \\
$16-19$ años & 37 & 27 & 51 & 115 \\
Mujeres & & & & \\
$12-15$ años & 47 & 46 & 48 & 141 \\
$16-19$ años & 51 & 41 & 49 & 141 \\
\hline Total & 166 & 165 & 200 & 531 \\
\hline
\end{tabular}

estar $\left(\mathrm{t}_{(525)}=-3,86 ; \mathrm{p}<0,001\right)$ y en resistencia $\left(\mathrm{t}_{(525)}=2,21 ; \mathrm{p}<0,05\right)$, a diferencia de las dimensiones riesgos y rendimiento, en donde el grupo de 13 a 15 años tiene medias superiores al grupo de mayor edad $\left(\mathrm{t}_{(525)}=2,90 ; \mathrm{p}<0,05 \mathrm{y} \mathrm{t}_{(525)}=2,07\right.$; $\mathrm{p}<0,05$ respectivamente).

La tabla 4 contiene los valores de referencia de los percentiles de las dimensiones del CHIP-AE según sexo y rango de edad, además del total. Se presenta asimismo los datos recogidos para similar población en el estudio español ${ }^{9}$. Tal como se puede observar, los valores encontrados para el total de hombres y mujeres para las diferentes dimensiones no varían mucho de lo reportado en el estudio realizado en Barcelona. Con excepción de la dimensión bienestar en los hombres, en donde los puntajes obtenidos en Chile son menor que los obtenidos para similar grupo en España, en tanto en las mujeres ocurre el fenómeno contrario, ya que tienen puntajes superiores a los de la muestra catalana.

La tabla 5 contiene los puntajes estandarizados para las distintas dimensiones y subdimensiones del CHIP-AE, estratificadas por sexo, comparándolas con el estudio español.

\section{Discusión}

El presente estudio muestra que los adolescentes varones escolarizados de la ciudad de Antofagasta presentan niveles de la satisfacción y resistencia significativamente más elevados, en tanto las mujeres puntúan más alto en bienestar. Es posible que este hecho se deba a diferencias basadas en construcciones de la salud diferenciadas por género, en donde los hombres centran su bienestar en la realización de actividades eminentemente físicas, con una menor atención a su salud y con menor conciencia de su cuerpo, en tanto, las mujeres priorizan actividades sociales o académicas, y con una mayor conciencia de su propio cuerpo y de los cambios que este sufre, disminuyendo 
Tabla 3. Puntajes estandarizados para dimensiones y subdimensiones del CHIPAE por grupos

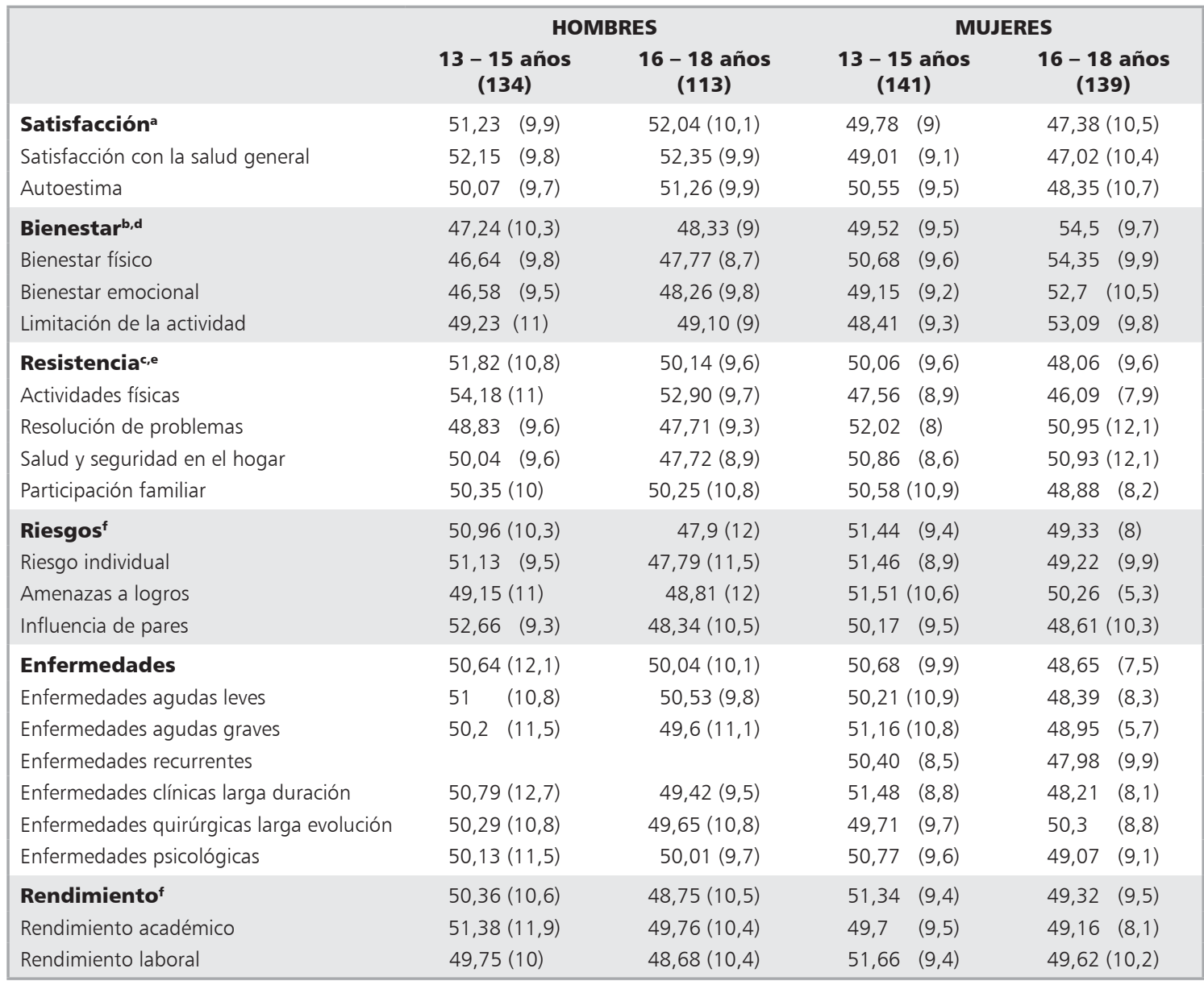

a Media de hombres significativamente mayor que mujeres $(\mathrm{p}<.01)$; ${ }^{b}$ Media de mujeres significativamente mayor que hombres $(p<.01)$; 'Media de hombres significativamente mayor que mujeres $(p<.05)$; dMedia de rango 16 a 18 años mayor que la de rango 13 a 15 años ( $<.01)$; eMedia de rango 16 a 18 años mayor que la de rango 13 a 15 años $(p<.05)$; ${ }^{\dagger}$ Media de rango 13 a 15 años mayor que la de rango 16 a18 años $(p<.05)$.

con esto la satisfacción con este. Se suma los patrones estéticos que comienzan a influir en la construcción del cuerpo femenino ideal, incluso en edades tempranas. El análisis por grupo etario reveló que los adolescentes entre 16 a 18 años tienen significativamente mayor puntuación en bienestar y en resistencia, a diferencia de las dimensiones riesgos y rendimiento, en donde el grupo de 13 a 15 años tiene medias superiores al grupo de mayor edad. Al comparar con el estudio español de Barcelona, los valores en las diferentes dimensiones no varían mucho excepto en bienestar, en donde los varones obtienen puntajes menores y las mujeres superiores a los de la muestra catalana.
Estudios longitudinales utilizando esta metodología, permitirán evaluar los cambios en la salud de adolescentes, percibida y evaluada desde su propia mirada, así como comparar sus resultados con otras poblaciones estudiadas.

Los datos generados por la utilización de este instrumento también posibilitan el diseño de programas específicos de intervención en salud adolescente, pudiendo constituirse en una herramienta diagnóstica adecuada en la evaluación de programas de salud o de efectos de intervenciones en prevención de la enfermedad o promoción de la salud.

La información presentada puede constituirse en un insumo importante para la eva- 
Tabla 4. Valores de referencia del perfil de salud CHIP AE a partir de una muestra de adolescentes escolarizados según sexo y rango de edad

\begin{tabular}{|c|c|c|c|c|c|c|c|c|}
\hline \multirow{2}{*}{$\begin{array}{l}\text { Deciles por } \\
\text { dimensión }\end{array}$} & & & & \multicolumn{2}{|c|}{ Total España } \\
\hline & $\begin{array}{c}13-15 \\
(134)\end{array}$ & $\begin{array}{c}16-18 \\
(113)\end{array}$ & $\begin{array}{c}13-15 \\
(141)\end{array}$ & $\begin{array}{c}16-18 \\
(139)\end{array}$ & $\begin{array}{l}\text { Hombres } \\
\text { (247) }\end{array}$ & $\begin{array}{c}\text { Mujeres } \\
\text { (280) }\end{array}$ & $\begin{array}{c}\text { Hombres } \\
\text { (475) }\end{array}$ & $\begin{array}{c}\text { Mujeres } \\
\text { (426) }\end{array}$ \\
\hline \multicolumn{9}{|l|}{ Satisfacción } \\
\hline P10 & 36,93 & 38,84 & 36,09 & 31,97 & 38,02 & 34,01 & 39,94 & 34,02 \\
\hline P20 & 43,67 & 44,58 & 40,97 & 38,33 & 44,10 & 40,11 & 45,22 & 38,76 \\
\hline P30 & 46,97 & 47,53 & 46,21 & 42,40 & 47,18 & 44,51 & 47,87 & 42,90 \\
\hline P40 & 49,26 & 51,04 & 48,75 & 46,21 & 50,58 & 47,48 & 50,80 & 46,01 \\
\hline P50 & 53,07 & 54,09 & 51,04 & 47,80 & 53,58 & 49,77 & 53,45 & 48,45 \\
\hline P60 & 55,36 & 56,33 & 53,37 & 51,04 & 55,87 & 52,31 & 55,50 & 50,86 \\
\hline P70 & 57,40 & 58,41 & 55,21 & 53,84 & 57,80 & 55,11 & 57,85 & 53,45 \\
\hline P80 & 59,68 & 60,44 & 57,45 & 56,38 & 60,19 & 57,04 & 59,91 & 55,79 \\
\hline P90 & 62,86 & 62,53 & 60,40 & 60,06 & 62,73 & 60,45 & 64,31 & 59,32 \\
\hline \multicolumn{9}{|l|}{ Bienestar } \\
\hline P10 & 37,59 & 38,46 & 39,65 & 43,08 & 37,89 & 40,73 & 40,47 & 33,21 \\
\hline P20 & 39,81 & 40,59 & 41,80 & 46,57 & 40,31 & 43,47 & 45,70 & 38,81 \\
\hline P30 & 41,50 & 42,86 & 43,51 & 48,90 & 42,04 & 46,06 & 48,44 & 43,18 \\
\hline P40 & 43,22 & 44,63 & 44,49 & 51,45 & 43,91 & 48,28 & 51,91 & 45,91 \\
\hline P50 & 45,16 & 46,99 & 47,20 & 52,87 & 45,94 & 51,42 & 53,64 & 49,00 \\
\hline P60 & 46,61 & 48,88 & 51,27 & 56,21 & 47,96 & 53,54 & 55,60 & 51,48 \\
\hline P70 & 49,35 & 51,31 & 54,26 & 57,64 & 50,03 & 56,21 & 57,85 & 53,82 \\
\hline P80 & 52,97 & 55,40 & 56,82 & 61,72 & 53,63 & 58,80 & 60,14 & 56,15 \\
\hline P90 & 59,24 & 61,74 & 61,41 & 66,54 & 59,64 & 65,03 & 62,89 & 59,11 \\
\hline \multicolumn{9}{|l|}{ Resistencia } \\
\hline P10 & 38,96 & 38,63 & 37,03 & 34,70 & 39,06 & 37,01 & 37,33 & 36,31 \\
\hline P20 & 42,87 & 42,80 & 41,99 & 41,13 & 42,88 & 41,60 & 43,18 & 41,32 \\
\hline P30 & 46,82 & 44,38 & 45,36 & 44,14 & 45,53 & 44,46 & 46,58 & 44,32 \\
\hline P40 & 49,70 & 48,62 & 48,70 & 46,70 & 49,15 & 48,35 & 49,76 & 47,32 \\
\hline P50 & 52,63 & 50,45 & 50,59 & 49,03 & 51,40 & 49,83 & 52,56 & 49,47 \\
\hline P60 & 54,76 & 52,08 & 52,31 & 50,59 & 53,58 & 51,65 & 54,44 & 51,30 \\
\hline P70 & 56,53 & 53,85 & 54,71 & 53,20 & 55,54 & 53,86 & 56,69 & 53,39 \\
\hline P80 & 59,52 & 56,74 & 56,41 & 54,91 & 58,86 & 55,69 & 60,13 & 55,33 \\
\hline P90 & 65,24 & 61,01 & 60,21 & 58,99 & 64,69 & 59,59 & 64,73 & 58,55 \\
\hline \multicolumn{9}{|l|}{ Riesgos } \\
\hline P10 & 36,54 & 29,20 & 39,96 & 39,92 & 33,24 & 39,96 & 35,35 & 37,42 \\
\hline P20 & 43,03 & 40,37 & 44,79 & 42,90 & 42,33 & 43,73 & 41,42 & 42,61 \\
\hline P30 & 47,33 & 43,84 & 48,38 & 45,04 & 46,03 & 46,55 & 45,22 & 45,30 \\
\hline P40 & 49,83 & 46,84 & 50,64 & 47,38 & 48,23 & 49,08 & 47,63 & 47,64 \\
\hline P50 & 52,05 & 48,89 & 52,44 & 50,32 & 50,69 & 51,39 & 50,16 & 50,28 \\
\hline P60 & 55,47 & 52,15 & 54,32 & 52,34 & 53,41 & 53,03 & 53,27 & 52,94 \\
\hline P70 & 57,11 & 54,87 & 56,15 & 54,07 & 55,92 & 55,52 & 56,43 & 56,83 \\
\hline P80 & 59,13 & 53,35 & 58,43 & 56,63 & 57,77 & 57,74 & 59,70 & 60,00 \\
\hline P90 & 60,96 & 59,22 & 60,42 & 58,57 & 60,67 & 59,58 & 62,52 & 63,11 \\
\hline \multicolumn{9}{|c|}{ Funcionamiento } \\
\hline P10 & 36,07 & 35,77 & 36,63 & 35,93 & 35,93 & 36,23 & 34,44 & 37,85 \\
\hline P20 & 38,35 & 37,81 & 41,78 & 39,69 & 38,35 & 39,74 & 41,49 & 45,31 \\
\hline P30 & 41,57 & 39,69 & 52,57 & 41,30 & 40,76 & 42,37 & 45,36 & 47,68 \\
\hline P40 & 53,11 & 43,23 & 54,18 & 53,11 & 52,04 & 54,18 & 47,68 & 49,23 \\
\hline P50 & 54,71 & 53,11 & 54,72 & 54,18 & 54,18 & 54,72 & 49,23 & 52,33 \\
\hline P60 & 55,26 & 54,72 & 55,26 & 54,72 & 55,23 & 55,26 & 52,33 & 53,88 \\
\hline P70 & 57,97 & 55,47 & 56,33 & 55,26 & 55,79 & 55,79 & 53,88 & 55,42 \\
\hline P80 & 57,40 & 56,44 & 56,87 & 56,33 & 56,87 & 56,87 & 56,97 & 58,52 \\
\hline P90 & 58,48 & 57,94 & 57,94 & 57,94 & 58,48 & 57,94 & 61,62 & 60,92 \\
\hline
\end{tabular}


Tabla 5. Puntajes estandarizados para dimensiones y subdimensiones del CHIPAE por sexo para Chile y España

\begin{tabular}{|c|c|c|c|c|}
\hline & \multicolumn{2}{|c|}{ Chile } & \multicolumn{2}{|c|}{ España } \\
\hline & $\begin{array}{l}\text { Hombres } \\
\text { (247) }\end{array}$ & $\begin{array}{c}\text { Mujeres } \\
(280)\end{array}$ & $\begin{array}{c}\text { Hombres } \\
\text { (475) }\end{array}$ & $\begin{array}{c}\text { Mujeres } \\
(\mathbf{4 2 6})\end{array}$ \\
\hline Satisfacción & 51,59 & 48,59 & 52,35 & 47,38 \\
\hline Satisfacción con la salud general & 52,24 & 48,02 & 53,01 & 46,64 \\
\hline Autoestima & 50,61 & 49,45 & 51,07 & 48,80 \\
\hline Bienestar & 47,74 & $51,99^{a}$ & $52,45^{b}$ & 47,36 \\
\hline Bienestar Físico & 47,16 & 52,50 & 52,24 & 47,50 \\
\hline Bienestar Emocional & 47,35 & 50,91 & 52,72 & 46,96 \\
\hline Limitación de la actividad & 49,17 & 50,73 & 50,45 & 49,49 \\
\hline Resistencia & 51,05 & 49,07 & 51,47 & 48,35 \\
\hline Actividades físicas & 53,59 & 46,82 & 54,22 & 45,29 \\
\hline Resolución de problemas & 48,32 & 51,49 & 48,15 & 52,06 \\
\hline Salud y seguridad en el hogar & 48,98 & 50,90 & 48,58 & 50,05 \\
\hline Participación familiar & 50,30 & 49,73 & 49,56 & 50,49 \\
\hline Riesgos & 49,56 & 50,39 & 49,61 & 50,43 \\
\hline Riesgo individual & 49,59 & 50,35 & 49,96 & 50,03 \\
\hline Amenazas a logros & 48,99 & 50,89 & 48,56 & 51,60 \\
\hline Influencia de pares & 50,68 & 49,39 & 50,43 & 49,52 \\
\hline Enfermedades & 50,37 & 49,67 & & \\
\hline Enfermedades agudas leves & 50,78 & 49,30 & & \\
\hline Enfermedades agudas graves & 49,93 & 50,06 & & \\
\hline Enfermedades recurrentes & & 49,19 & & \\
\hline Enfermedades clínicas larga duración & 50,16 & 49,85 & & \\
\hline Enfermedades quirúrgicas larga evolución & 49,99 & 50,00 & & \\
\hline Enfermedades Psicológicas & 50,08 & 49,92 & & \\
\hline Rendimiento & 49,62 & 50,34 & 48,76 & 51,28 \\
\hline Rendimiento académico & 50,64 & 49,43 & 49,21 & 50,88 \\
\hline Rendimiento laboral & 49,26 & 50,65 & 49,27 & 50,71 \\
\hline
\end{tabular}

${ }^{a}$ Media significativamente mayor que la de las mujeres españolas $(p<.01)$; ${ }^{b}$ Media significativamente mayor que la de los hombres chilenos $(p>.01)$.

luación pediátrica centrada en factores no mórbidos, que le permitirían al clínico contar con una mirada más comprensiva de la salud del adolescente, brindándole la posibilidad de comparar los resultados obtenidos por el adolescente con otros datos de similar población. Su uso a nivel de salud pública, nos permite focalizar posibles áreas en detrimento o riesgo que ameriten la intervención mediante programas preventivos, en pro de la salud integral del adolescente y no solo de la búsqueda de enfermedades o trastornos, atendiendo además a las particularidades dadas por el género, en virtud de los valores reportados.

Asimismo, el poder contar con información percibida desde los propios adolescentes con- tribuye de alguna manera a disminuir la brecha percibida entre la oferta en salud y la demanda real de atención en salud adolescente ${ }^{19}$, favoreciendo el acercamiento de las intervenciones en salud pública a al mundo juvenil.

La comparación de los datos que podamos obtener con los valores presentados, abre la posibilidad de pesquisar adolescentes que puedan requerir atención sanitaria, ya que diferencias superiores a 0,5 DE se presentarían en adolescentes que requerirían atención sanitaria ${ }^{15}$.

Las limitaciones de este estudio están relacionadas con que solo se ha podido presentar valores de referencia de la ciudad de Antofagasta, dado que no ha sido posible realizar un muestreo representativo nacional. Por otra par- 
te, el estudio incorpora solamente adolescentes escolarizados, que aun cuando comprenden un promedio cercano al $91 \%{ }^{20}$, no representa la realidad en salud de 1 de cada 10 adolescentes que no están por diversos motivos, incorporados a la red educacional. Estas limitantes abren nuevas demandas de investigación en el campo de la salud adolescente que pueden aportar insumos a los nuevos desafíos en el campo de la salud pediátrica ${ }^{21}$.

\section{Conclusión}

El presente estudio demuestra que los adolescentes escolarizados de la ciudad de Antofagasta presentan un perfil similar al descrito para adolescentes españoles en la mayoría de las dimensiones, excepto en bienestar donde los varones obtienen puntajes menores y las mujeres superiores

Los datos que aporta este estudio, pueden ser utilizados como valores de referencia en adolescentes escolarizados de Antofagasta al utilizar el cuestionario CHIP-AE, generando la oportunidad de monitorear el comportamiento tanto de la salud de este grupo etario, como de los factores asociados a esta, permitiendo analizar los cambios en esta percepción de salud que permitan focalizar futuras intervenciones. Así mismo, permitirá comparar la autopercepción de salud y enfermedad de la población estudiada con otras poblaciones nacionales o extranjeras al utilizar el mismo instrumento.

\section{Agradecimientos}

A la Dra. V. Serra Sutton, quien proporcionó los valores del estudio realizado en la ciudad de Barcelona, los cuales no estaban publicados.

\section{Referencias}

1.- Martínez M: Mirando el futuro: desafíos y oportunidades para el desarrollo de los adolescentes en Chile. Psykhe 2007; 16 (1): 3-14.

2.- Eiser $C$, Morse R: Review of measures of quality of life in children with chronic illness. Arch Dis Child 2001; 84: 205-11.

3.- Harding L: Children's Quality of life assessment: a review of generic and health related quality of life measures completed by children and adolescents. Clin Psychol Psychoteraphy 2001; 8: 79-96.

4.- Rajmil L, Herdman M, Fernández $M$, et al \& The KIDSCREEN Group: Generic Health-related Quality of Life Instruments in Children and Adolescents: A Qualitative Análisis of Content. J Adolescent Health 2004; 34: 37-45.

5.- Rajmil L, Estrada M, Herdman M, Serra-Sutton V, Alonso $J$ : Calidad de vida relacionada con la salud (CVRS) en la infancia y la adolescencia: revisión de la bibliografía y de los instrumentos adaptados en España. Gac Sanit 2001; 15 (Supl 4): 34-43.

6.- Urzúa A \& Mercado G. La evaluación de la calidad de vida de los y las adolescentes a través del Kiddo- Kindl. Terapia Psicológica 2008; 26 (1): 133-41.

7.- Urzúa A, Cortés E, Prieto L, Tapia K, Vega S: Propiedades psicométricas del cuestionario de evaluación de la Calidad de Vida en niños y adolescentes KIDSCREEN-27. Terapia psicológica 2009; 27 (1): 83-92.

8.- Urzúa A, Méndez F, Acuña C, Astudillo Y: Calidad de Vida relacionada con la salud en edad preescolar. Rev Chil Pediatr 2010; 81 (2): 128-37.

9.- Serra-Sutton V, Rajmil L, Alonso J, Riley A, Starfield B: Valores poblacionales de referencia del perfil de salud CHIP-AE a partir de una muestra representativa de adolescentes escolarizados. Gac Sanit 2003; 17 (3): 181-9.

10.- Avendaño M, Barra E: Autoeficacia, apoyo social y Calidad de Vida en adolescentes con enfermedades crónicas. Terapia Psicológica 2008; 26 (2): 165-72.

11.- Urzúa A, Cortés E, Prieto L, Vega S: Autoreporte de la calidad de vida en niños y adolescentes escolarizados. Rev Chil Pediatr 2009; 80 (3): 238-44.

12.- Urzúa A, Avendaño F, Díaz S, Checura D: Calidad de Vida y conductas alimentarias en la preadolescencia. Revista Chilena de Nutrición 2010; 37 (3): 282-92.

13.- Starfield B, Bergner M, Ensminger M, et al: Adolescent health status measurement: development of the Child Health and Illness Profile. Pediatrics 1993; 91 (2): 4305.

14.- Starfield B, Riley A, Green B, et al: The adolescent Child health and illness profile: a population-based measure on health. Med Care 1995; 33 (5): 553-66.

15.- Rajmil L, Serra Sutton V, Alonso J, Starfield B, Ruley $A$, Vázquez $J$ \& the research group for the Spanish version of the CHIP-AE: The Spanish version of the Child Health and Illness Profile - Adolescent Edition 
URZÚA A. y cols.

(CHIP-AE). Quality of Life Res 2003; 12: 303-13.

16.- Rajmil L, Serra Sutton V, Alonso J, Herdman M, Riley A, Starfield B: Validity of the Spanish version of the Child health and illness profile- adolescent Edition (CHIP-AE). Med Care 2003; 41 (10): 1153-63.

17.- Pérez D, Blanco M, García R, Suárez R: Adaptación y ensayo de un cuestionario de calidad de vida relativa a salud en adolescentes. Rev Cubana Hig Epidemiol 2008; 46 (1).

18.- Urzúa A, Cárdenas M: Salud en la adolescencia: propiedades psicométricas del perfil de salud y enfermedad (CHIP-AE) en adolescentes chilenos. Psicología: Re- flexao e Crítica 2011; 24 (2): 254-63.

19.- Berger C: Subjetividad adolescente: tendiendo puentes entre la oferta y demanda de apoyo psicosocial para jóvenes. Psykhe 2004; 13 (2): 143-57.

20.- UNICEF (2010). Sitio web oficial de United Nations Children's Fund , organismo dependiente de la Organización de las Naciones Unidas, disponible en website: http://www.unicef.org/spanish/infobycountry/chile_statistics.html\#67, extraido el 14 de diciembre de 2010.

21.- Vargas N, Quezada A: Epidemiología, nueva morbilidad pediátrica y rol del pediatra. Rev Chil Pediatr 2007; 78 (supl 1): 103-10. 